\title{
Productive Cough and Bilateral Ptosis
}

\author{
Van Den Bergh $\mathrm{A}^{* 1}$, Ninane $\mathrm{V}^{2}$, Yserbyt $\mathrm{J}^{1}$, Lorent $\mathrm{N}^{1}$, Dooms $\mathrm{C}^{1}$ \\ ${ }^{1}$ University Hospital Leuven-Department of Respiratory Medicine, Leuven, Belgium \\ ${ }^{2}$ St. Pierre Hospital, Brussels, Belgium
}

${ }^{*}$ Corresponding author: Van Den Bergh A, MD, University Hospital Leuven-Department of Respiratory Medicine, Leuven, Belgium, Tel: +32479772868, E-mail: annelies.vandenbergh@uzleuven.be

Citation: Van Den Bergh A, Ninane V, Yserbyt J, Lorent N, Dooms C (2018) Productive Cough and Bilateral Ptosis. J Case Rep Stud 6(4): 403. doi: 10.15744/2348-9820.6.403

Received Date: May 17, 2018 Accepted Date: August 21, 2018 Published Date: August 23, 2018

\begin{abstract}
We present a case report of a patient with Mounier-Kuhn syndrome. The clinical, radiological and endoscopic illustrations contribute to the awareness and recognizability of this rare and under-diagnosed pulmonary syndrome. It is characterized by tracheobronchomegaly and tracheomalacia, leading to ineffective mucociliary clearance, recurrent respiratory infections and bronchiectasis. Computed tomography is the golden standard for diagnosis. Etiology remains unknown. Prognosis depends on adequate treatment and prevention of respiratory infections.
\end{abstract}

Keywords: Bronchiectasis; Endoscopy; Connective Tissue Disease

\section{Introduction}

Mounier-Kuhn syndrome is a rare syndrome mostly affecting adult males (8:1) and smokers [1]. The prevalence is unknown, but publications estimate between 0.4 and $1.6 \%$ in patients with respiratory symptoms [2]. Mounier-Kuhn Syndrome is defined by tracheobronchomegaly (dilatation of the trachea and main airways) and tracheomalacia (tracheal collapse) leading to ineffective mucociliary clearance, recurrent pulmonary infections and secondary bronchiectasis. Since an enlarged trachea is easily overlooked on chest X-ray, CT thorax is the golden standard for diagnosis [3]. Histopathological, thinning of muscular mucosa and atrophy of longitudinal muscle and elastic fibers contribute to the diagnosis [2]. This syndrome has been associated with connective-tissue diseases, such as Ehlers-Danlos or cutis laxa [2].

We believe that this case presentation and its clinical, radiological and endoscopic illustrations contribute to the increasing awareness and recognizability of this rare syndrome.

\section{Case report}

A 61-year-old man presented at the emergency department with fever and a productive cough since one week, despite treatment with amoxicillin/clavulanic acid. He was an ex-smoker (20 pack-years). His medical history included bronchiectasis and Chronic Obstructive Pulmonary Disease Gold grade 2, group C [4]. At presentation, positive inspection and examination signs included elevated temperature $\left(38.2{ }^{\circ} \mathrm{C}\right)$, low transcutaneous oxygen saturation $(90 \%$ breathing room air) and bilateral ptosis (Figure 1$)$. Lung auscultation revealed crackles at both lung bases. Laboratory investigations included a leukocytosis $12.5 \mathrm{x} 10^{\star} 9 / \mathrm{L}(82 \%$ neutrophils) and an elevated C-reactive protein $(180 \mathrm{mg} / \mathrm{L})$. Chest X-ray demonstrated hazy consolidations peribronchial in the right lower lobe as well as a limited pleurisy ipsilateral. CT thorax confirmed bronchiectasis in both lower lobes with tree-inbud, consistent with infection. It also revealed a striking tracheobronchomegaly (Figure 2). Flexible bronchoscopy confirmed tracheobronchomegaly with circumferential mucosal folds from the subglottis region to the subsegmental bronchi, in the absence of the pars membranacea (Figure 3).

Microbiological examination indicated the presence of P. aeruginosa and E. cloacae, with a favorable clinical response to piperacillin/ tazobactam. Further work-up for bronchiectasis revealed no immunodeficiency nor arguments for allergic bronchopulmonary aspergillosis, primary ciliary dyskinesia, cystic fibrosis or any mixed connective tissue disease or rheumatoid arthritis. Unfortunately, no positive arguments could confirm the diagnosis histopathological. A sampling error could possibly account for that, although all biopties were taken in the posterior tracheal wall at the mucosal folds. The patient was discharged in good health after 2 weeks IV antibiotics. Hypertonic saline inhalation and physiotherapy were added to his maintenance therapy. 


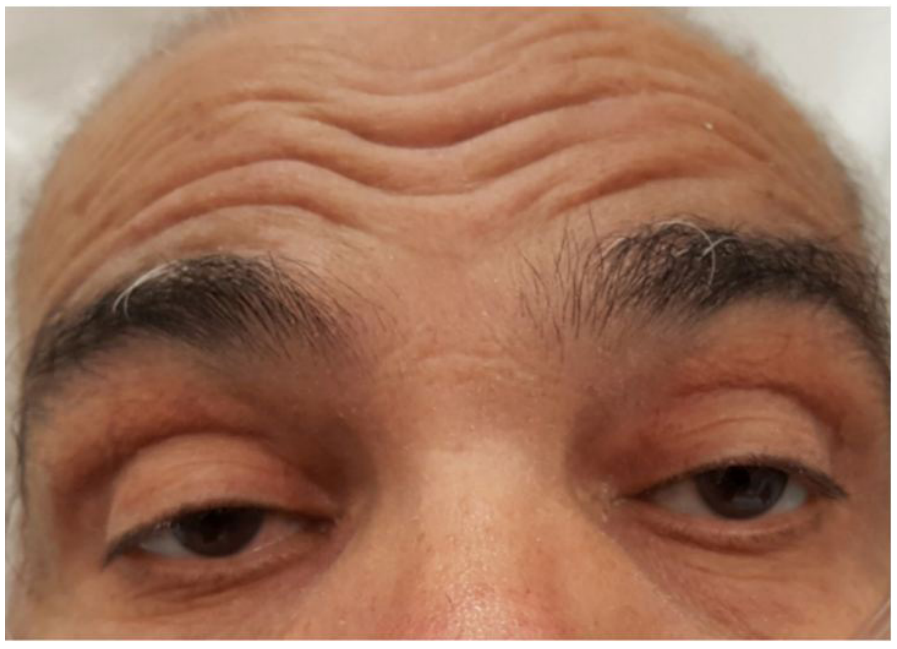

Figure 1: Bilateral ptosis interpreted as mark of cutis laxa. Note the frontalis muscle contraction

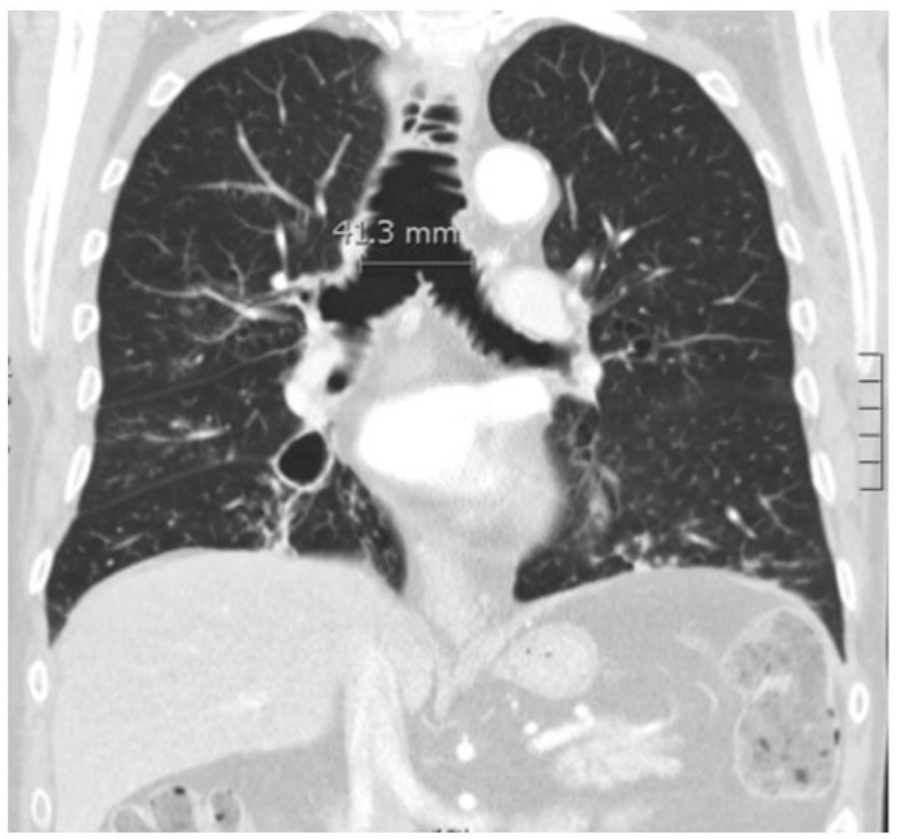

Figure 2: Computed tomography showing increased tracheal diameter (at coronal reconstruction)

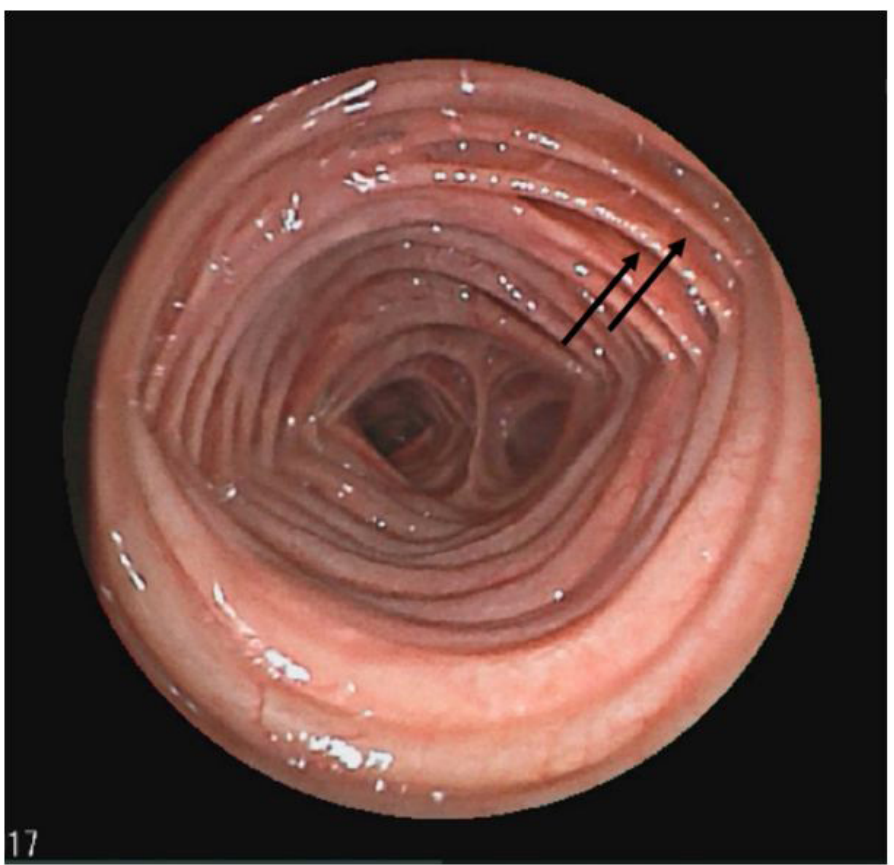

Figure 3: Endoscopic view of the trachea with absence of the pars membranacea and presence of diverticula (arrows) 


\section{Discussion}

Mostly adult males and smokers are affected by the syndrome, but it also occurs in children as well as in the geriatric population [2,3]. Hereby, the pathophysiology of Mounier-Kuhn Syndrome remains subject of debate. Thinning of muscular mucosa and atrophy of longitudinal muscles and elastic fibers are the histopathological correlate for the tracheal mucosal folds separated by saccular diverticula [2]. Whether this is due to a congenital disorder (explaining its appearance in toddlers and its association with other connective tissue diseases) or due to an underlying inflammation, remains unclear. However, a thorough investigation should exclude secondary causes of tracheobronchomegaly.

Signs and symptoms are not specific. Infection, chronic cough and dyspnea are the most common, but Mounier-Kuhn Syndrome may also be asymptomatic [5]. Evolution and prognosis depend on adequate treatment and prevention of respiratory infections. Airway clearance techniques and hyperosmolar agents are the cornerstone of patient's maintenance treatment, as it is for all non-CF bronchiectasis. General anesthesia in Mounier-Kuhn Sydrome can be challenging because of significant air loss during ventilation and a high aspiration risk [2,6]. Extensive preoperative evaluation is necessary to explore the best approach for general anesthesia.

\section{References}

1. Krustins E (2016) Mounier-Kuhn syndrome: a systematic analysis of 128 cases published within last 25 years: Congenital tracheobronchomegaly - statistical review. Clin Respir J 10: 3-10.

2. Krustins E, Kravale Z, Buls A (2013) Mounier-Kuhn syndrome or congenital tracheobronchomegaly: A literature review. Respir Med 107: 1822-8.

3. Akgedik R, Karamanli H, Kizilirmak D, Kurt AB, Öztürk H, et al. (2018) Mounier-Kuhn syndrome (tracheobronchomegaly): An analysis of eleven cases. Clin Respir J 12: 885-9.

4. Marçôa R, Rodrigues DM, Dias M, Ladeira I, Vaz AP, et al. (2018) Classification of Chronic Obstructive Pulmonary Disease (COPD) according to the new Global Initiative for Chronic Obstructive Lung Disease (GOLD) 2017: Comparison with GOLD 2011. COPD 15: 21-6.

5. Schmitt P, Dalar L, Jouneau S, Toublanc B, Camuset J, et al. (2016) Respiratory Conditions Associated with Tracheobronchomegaly (Mounier-Kuhn Syndrome): A Study of Seventeen Cases. Respiration 91: 281-7.

6. Lee C-C, Lin B-S, Chen J-Y, Chuang C-C (2017) Anesthesia for a patient with unexpected giant tracheobronchomegaly. Tzu Chi Med J 29: 59.

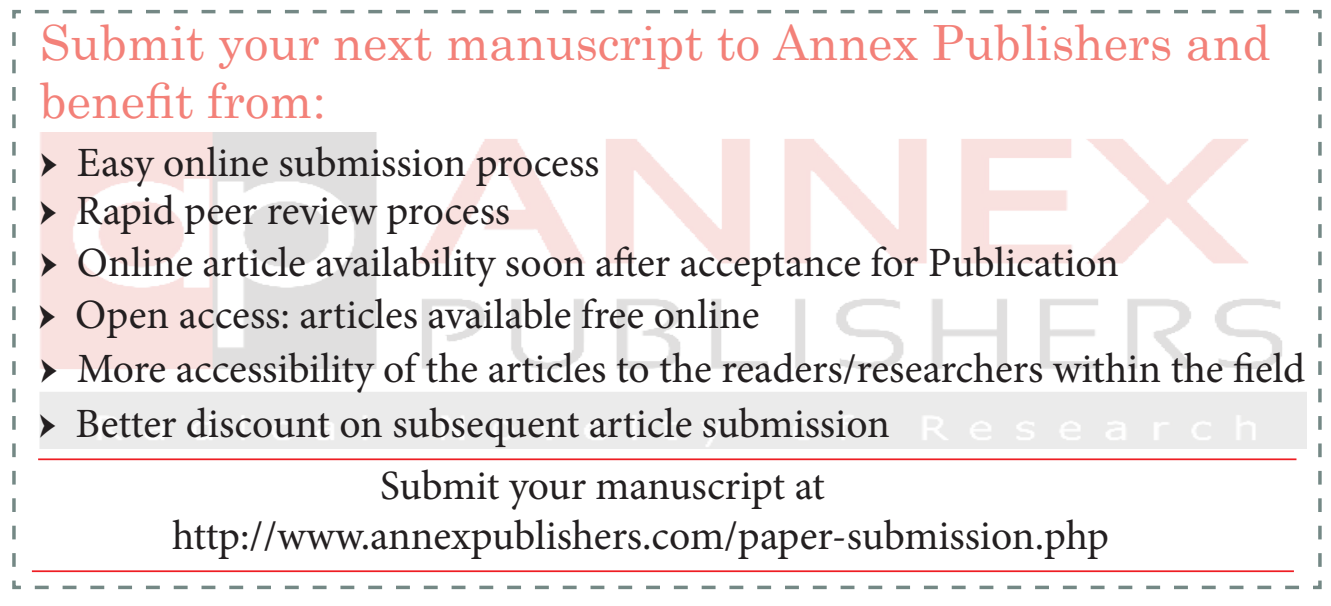

